\title{
ANÁLISE DE SOBRECARGAS ADQUIRIDAS POR CUIDADORES E SEUS PACIENTES COM COMPLICAÇÕES NEUROLÓGICAS ANTES E APÓS UM PROGRAMA DE ORIENTAÇÕES
}

\author{
ANALYSIS OVERLOADS ACQUIRED \\ FOR CAREGIVERS AND THIR \\ PATIENTS WITH NEUROLOGICAL \\ COMPLICATIONS BEFORE AND \\ AFTER GUIDANCE PROGRAM.
}

Régis Inocêncio Valério da Luz* Tiago Tsunoda Del Antonio** Camila Costa de Araújo *** Ana Paula do Nascimento**** Marcos da Cunha Lopes Virmond $d^{* * * * *}$ Mirella Lindoso Gomes Campos ${ }^{* * * * * *}$ Joyce Karla Machado da Silva***

RESUMO

Cuidador é o indivíduo que oferece suporte a uma pessoa cuidada, mas muitos iniciam seus papéis com poucas ou sem instruções. O projeto "Funcionalidade e Qualidade de Vida de Pacientes com Complicações Neurológicas e Cuidadores (familiares)" esteve vinculado ao Programa Institucional de Bolsas de Extensão Universitária. Participaram cuidadores e pacientes, atendidos na Clínica de Fisioterapia da Universidade Estadual do Norte do Paraná, que foram submetidos à avaliação inicial e final, como também à intervenção de 10 semanas sobre cuidados com pacientes a domicílio. Com o objetivo de "Avaliar a Qualidade de Vida, autoestima e contato com a Síndrome de Burnout desses pacientes e cuidadores, após um programa de orientações", constatou-se o comprometimento de qualidade de vida; a autoestima apresentou escore médio antes e após orientações (15,5 para 14 nos cuidadores e 16,3 para 15,5 nos pacientes) e notou-se menor influência da Burnout após a intervenção, diminuindo $6,7 \%$ da pontuação.

Palavras-chave: cuidador; orientações; pacientes; neurologia.

\footnotetext{
* Aluno de graduação da Universidade Estadual do Norte do Paraná (UENP), PR - Brasil. Email: regis_luz_@hotmail.com

** Professora da Universidade Estadual do Norte do Paraná (UENP), PR - Brasil. Email: tiagodantonio@uenp.edu.br

*** Professora da Universidade Estadual do Norte do Paraná (UENP), PR - Brasil. Aluna de Doutorado da Universidade do Sagrado Coração (USC), SP - Brasil. Email: camilaaraujo@uenp.edu.br

**** Aluna de graduação da Universidade Estadual do Norte do Paraná (UENP), PR - Brasil. Email: anaapaulanascimento@gmail.com

***** Professor da Universidade do Sagrado Coração (USC), SP - Brasil. Email: mvirmond@ilsl.br;

****** Professora da Universidade do Sagrado Coração (USC), SP - Brasil. Email: mirellalindoso@gmail.com

******* Professora da Universidade Estadual do Norte do Paraná (UENP), PR - Brasil. Aluna de Doutorado da Universidade do Sagrado Coração (USC), SP - Brasil. Email: jksilva@uenp.edu.br
} 


\begin{abstract}
Caregivers support people in need, but most start working with little or no instruction. The project "Functionality and quality of life of patients with neurological complications and their caregivers (relatives)" was linked to the Institutional Program of University Scholarships. Four caregivers and patients assisted by the physiotherapy clinic of the State University of the North of Paraná underwent an initial and final evaluation and a 10-week program about patients' care at home. The study aimed at evaluating the quality of life, self-esteem and burnout syndrome of patients with neurological complications and their caregivers, before and after a guideline program. It was found that the quality of life was impaired even after the program, self-esteem presented a mean score before and after the orientations ( 15.5 to 14 for the caregivers and 16.3 to 15.5 for the patients), and a lower influence of burnout after the intervention, decreasing $6.7 \%$ of the score.
\end{abstract}

Keywords: caregiver; instructions; patients; neurology.

\title{
Introdução
}

O termo cuidador designa aquele indivíduo que assume a responsabilidade de prestar cuidados no contexto domiciliar e, na atividade dessa função, representa o elo entre o paciente, a família e a equipe multiprofissional (SCHNAIDER; SILVA; PEREIRA, 2009).

Considerado como um importante precursor de impacto positivo na vida de pacientes, em geral, ele faz parte de uma população que é aceita como ator-chave na prestação de cuidados e, em contrapartida, são frequentemente despercebidos (ALMUTAIRI et al, 2016).

Não obstante à atuação desses indivíduos, podem-se visualizar problemas de saúde física como fadiga, falta de apetite e sono, bem como, um maior risco de mortalidade e problemas mentais experimentados por essa população, em comparação aos não-cuidadores (ALMUTAIRI et al, 2016). Além disso, os cuidadores, principalmente os que são familiares, geralmente se deparam com situaçóes inusitadas e começam seus papéis sem treinamento, procurando atender grande demanda com raro apoio e assistência. Nesse sentido, esse quadro pode induzir à angústia, depressão e baixa qualidade de vida experimentada pelos cuidadores (ALMUTAIRI et al, 2016).

Frente a essas possíveis sobrecargas vem a necessidade, em especial dos profissionais de saúde, em observar como os cuidadores de pessoas com afecçóes neurológicas estão se comportando no desenvolvimento das suas atividades cotidianas (SCHNAIDER; SILVA; PEREIRA, 2009).

Atualmente, sabe-se que cuidar de um paciente com doença avançada em domicílio causa importante sobrecarga ao cuidador e a sua família, com prejuízo de qualidade de vida, autoestima e até a mesmo a possibilidade do desenvolvimento da Sindrome de Burnout, apresentada pelos cuidadores (FLORINI, 2004).

O conceito "qualidade de vida" se conecta com o estado de bem estar geral, envolvendo tanto aspectos físicos, psíquicos, como também, sociais. A "qualidade de vida é uma noção humana que se interliga ao grau de satisfação encontrado na vida familiar, amorosa, social e ambiental e até mesmo à própria existência” (FERNANDES; VASCONCELOS; SILVA, 2009).

Por autoestima é entendido a avaliação que o indivíduo faz de si mesmo. É expressa como uma atitude de aprovação ou de negação de si e engloba o autojulgamento em relação à competência e valor. É o juízo pessoal de valor revelado através das atitudes de um indivíduo com si próprio, sendo uma experiência subjetiva, acessível às pessoas através de relatos verbais e comportamentos observáveis (AVANCI et al, 2007). Pode ser avaliada segundo os seguintes níveis: baixa autoestima: caracterizada pelo sentimento de incompetência, de inadequação à vida e incapacidade de superar desafios; média autoestima: se interliga entre o sentimento de adequação ou inadequação, manifestando essa inconsistência no comportamento, 
já a alta autoestima expressa um sentimento de confiança, como também, de competência (AVANCI et al, 2007).

Ainda visando às sobrecargas impostas a esta função, é possível averiguar o contato da Síndrome de Burnout com essa populaçáo. No linguajar popular inglês, o termo significa aquilo que deixou de funcionar por absoluta falta de energia. Primeiramente, o termo foi utilizado no ano de 1974, por Freudenberger, descrevendo o mesmo como um sentimento de fracasso e exaustáo causada por um excessivo desgaste de energia e recursos (PAGANINI, 2011). Esta síndrome tem como sintomas iniciais a exaustáo emocional, e logo após sentimentos e atitudes negativas e, insensibilidade afetiva. Por fim, é manifestado sentimento de insatisfaçáo pessoal e no trabalho, prejudicando sua eficiência e habilidade para a realização de tarefas (SILVA; BRAGA; SILVA, 2009).

Diante de toda essa problemática expressa na relação cuidador/paciente, o estudo teve como objetivo avaliar a Qualidade de Vida e aspectos relacionados (Autoestima e Sindrome de Burnout) de pacientes com complicaçôes neurológicas e seus cuidadores, antes e após um programa de orientaçóes.

\section{Métodos}

O presente projeto foi intitulado "Funcionalidade e Qualidade de Vida de Pacientes com Complicaçôes Neurológicas e Seus Cuidadores (familiares)" e participaram da pesquisa 6 cuidadores e seus respectivos pacientes neurológicos atendidos na Clínica de Fisioterapia Prof. Alfredo Franco Ayub, da Universidade Estadual do Norte do Paraná UENP, Centro de Ciências da Saúde - CCS. Porém, durante o decorrer das atividades, por motivos diversos, apenas 4 cuidadores e seus respectivos pacientes continuaram até a finalização do trabalho. $\mathrm{O}$ projeto teve a Fundação Araucária como órgáo de fomento, fazendo parte do Programa Institucional de Bolsas de Extensão Universitária (PIBEX).

Como critérios de inclusão utilizaram-se pacientes e seus respectivos cuidadores que estavam em tratamento na clínica já citada, com diagnóstico de lesão neurológica. Foi dividido em três fases o projeto: na primeira foram realizadas as avaliaçóes iniciais utilizando as escalas SF-36 para investigar a Qualidade de Vida dos cuidadores e pacientes, Escala de Autoestima de Rosenberg (RSES) para aferir a autoestima de todos os participantes e o Questionário Preliminar de Identificação da Burnout (MBI), para averiguar o contato da Sindrome de Burnout com os cuidadores. Na segunda fase foi oferecido aos cuidadores/pacientes um programa de orientaçóes referente aos cuidados básicos prestados pelos cuidadores e, na terceira fase com avaliaçóes finais, sendo utilizados os mesmos questionários das avaliaçóes iniciais.

O SF-36 avalia mediante 8 domínios: capacidade funcional, limitação por aspectos físicos, dor, estado geral de saúde, vitalidade, aspectos sociais, aspectos emocionais e saúde mental. Ele apresenta um escore final em cada domínio que vai de zero a 100 pontos, no qual zero corresponde ao pior estado geral de saúde e 100 ao melhor estado de saúde (TSUKAMOTO; PICINATTO; CAVALINI; BORTOLLOTI, 2010).

A RSES foi desenvolvida a partir das pontuaçôes de 5024 participantes de ambos os sexos, incluindo estudantes universitários e pessoas adultas, provenientes de meios sociais diferentes e grupos étnicos diversificados. Originalmente, concebida como escala Guttman, o RSES também pode ser cotada simplesmente somando os itens tipo Likert de 
4 pontos $($ Discordo fortemente $=0$, Discordo $=1$, Concordo $=2$, Concordo fortemente $=$ $3)$, após se ter feito a reversão dos pontos, a pontuação na escala varia entre 0 e 30 , sendo que pontuaçóes elevadas na escala indicam autoestima elevada e vice-versa (PECHORRO et al, 2011).

O teste MBI é um questionário preliminar de identificação da Burnout, que já foi validado pelo Núcleo de Pesquisas Avançadas sobre a Sindrome de Burnout, pelo Departamento de Psicologia da Universidade Estadual de Maringá, e sofreu algumas adaptaçôes de terminologia para melhor direcionar os grupos pesquisados. É o instrumento mais utilizado nas pesquisas sobre a síndrome, chegando a ser considerado uma verdadeira tautologia com equivalência entre o constructo e seus instrumentos de medida, isto é, o "Burnout é o que se mede com o MBI" (SILVA; BRAGA; SILVA, 2009).

$\mathrm{Na}$ segunda fase, as orientaçóes aconteceram a domicílio, sendo realizado encontros semanais com duração em média de 40 minutos a 1 hora cada sessão, tendo duraçáo total o acompanhamento de 10 semanas com cada cuidador/paciente. As orientaçóes tiveram como base uma cartilha explicativa, sendo abordados diversos temas sobre cuidados gerais prestados pelos cuidadores, entre eles, transferências sendo realizada por um e dois cuidadores, e até mesmo quando possível as transferências individuas; mudanças de decúbito dorsal para laterais, ventral e vice versa; posicionamento adequado no leito utilizando alguns recursos para melhor conforto como travesseiros e almofadas; prevençóes de úlceras de pressão trabalhando posicionamentos e mudanças de decúbito; higiene com o paciente, incluindo banhos e meios seguros de realizá-los.

Além disso, foram realizadas orientaçóes gerais, tanto para os pacientes como para os cuidadores, buscando atender às necessidades isoladas, como por exemplo, exercícios de alongamentos individuais ou em duplas, e exercícios funcionais para os pacientes, a fim de tratar seus déficits de acordo com seus diagnósticos.

\section{Resultados}

De acordo com a aplicaçáo da Escala SF-36 nos cuidadores, pode-se notar que, inicialmente, antes do programa de orientaçóes, as médias com menores valores dos domínios obtidos foram: $1^{\circ}$ Estado Geral de Saúde com 56,5 de score; 20 Dor com 69,5 de pontuaçáo e $3^{\circ}$ Vitalidade com 71,25 de média. Após o programa de orientaçôes os piores escores obtidos foram: $1^{\circ}$ Estado Geral de Saúde com 59,5 de pontuação; $2^{\circ}$ Vitalidade com 71,25 e $3^{\circ}$ Dor com 75 de média final. Resultados apresentados na tabela1.

Tabela 1. Médias iniciais e finais obtidas na aplicação do SF-36 aos cuidadores estudados.

\begin{tabular}{l|l|l}
\hline & SF - 36 Inicial & SF - 36 Final \\
\hline Capacidade Funcional & 92,5 & 86,25 \\
\hline Aspecto Físico & 93,75 & 81,25 \\
\hline Dor & 69,5 & 75 \\
\hline Estado Geral de Saúde & 56,5 & 59,5 \\
\hline Vitalidade & 71,25 & 71,25 \\
\hline Aspectos Sociais & 93,75 & 91,75 \\
\hline Aspectos Emocionais & 91,75 & 100 \\
\hline Saúde Mental & 85 & 78
\end{tabular}


Em relação à aplicação do SF-36 nos pacientes, os piores escores obtidos anteriores ao programa de orientaçōes foram: $1^{\circ}$ Capacidade Funcional obtendo 0 de escore; $2^{\circ}$ Estado Geral de Saúde com 59,65 de média e $3^{\circ}$ Vitalidade com 73,3. Após o programa de orientaçôes: $1^{\circ}$ Capacidade Funcional com 0 de escore; $2^{\circ}$ Estado Geral de Saúde com 52 pontos e $3^{\circ}$ Vitalidade com 65 de média. Resultados apresentados na tabela 2.

Tabela 2. Médias iniciais e finais obtidas na aplicação do SF-36 aos pacientes estudados.

\begin{tabular}{l|l|l}
\hline & SF - 36 Inicial & SF - 36 Final \\
\hline Capacidade Funcional & 0 & 0 \\
\hline Aspecto Físico & 91,65 & 75 \\
\hline Dor & 74,3 & 74,3 \\
\hline Estado Geral de Saúde & 59,65 & 52 \\
\hline Vitalidade & 73,3 & 65 \\
\hline Aspectos Sociais & 100 & 100 \\
\hline Aspectos Emocionais & 100 & 100 \\
\hline Saúde Mental & 78,65 & 78,65
\end{tabular}

Em relação aos resultados da aplicação da Escala de Rosenberg foi percebida uma diminuição nas médias finais obtidas em ambos os grupos de indivíduos avaliados. Resultados Explanados na tabela 3.

Tabela 3. Escores iniciais e finais obtidos a partir da aplicação da Escala de Rosenberg.

\begin{tabular}{l|l|l}
\hline & Rosenberg Inicial & Rosenberg Final \\
\hline & & \\
\hline Cuidadores & 15,5 & 14 \\
\hline Pacientes & 16,3 & 15,5 \\
\hline
\end{tabular}

Quanto à Burnout, foi apontada uma diminuição do contato dos cuidadores com a síndrome, sendo que na aplicação da MBI inicial, os cuidadores obtiverem uma pontuação de 49,75 de média e final de 43 conforme a tabela 4 .

Tabela 4. Pontuação da MBI antes e após o programa de orientações.

\begin{tabular}{l|l|l}
\hline & Burnout Inicial & Burnout Final \\
\hline Cuidadores & 49,75 & 43 \\
\hline
\end{tabular}

\section{Conclusão}

O projeto teve grande contribuiçáo social com os voluntários envolvidos. Ainda que a qualidade de vida e a autoestima sustentaram seus resultados, tanto iniciais como finais, após a intervenção, o programa de orientaçóes foi essencial para diminuição da influência da Sindrome Burnout na vida do grupo de cuidadores, sendo que as instruçôes contribuíram para um melhor manejo e confiança durante a prática de suas atividades e, consequentemente, maior confiança dos pacientes no recebimento desses cuidados. Embora o projeto tenha acontecido com um número reduzido de participantes, a pesquisa continua com perspectiva de ampliaçáo do estudo alcançando um maior número de indivíduos. 


\section{Referências}

ALMUTAIRI, K. M.; ALODHAYANI, A. A.; ALONAZY, W. B.; VINLUAN, J. M. Assessment of Health-Related Quality of Life Among Caregivers of Patients with Cancer Diagnosis: A Cross-Sectional Study in Saudi Arabia. New York: J Relig Health, 2016.

AVANCI, J. Q.; ASSIS, S. G.; SANTOS, N. C.; OLIVEIRA, R. V. C. Adaptação Transcultural de Escala de Auto-Estima para Adolescentes. Psicologia: Reflexão e Crítica, Porto Alefre, v. 20, n. 3, p. 397-405, 2007. Disponível em: <http://www.scielo.br/pdf/prc/v20n3/a07v20n3.pdf>. Acesso em: 10 dez. 2016.

FERNANDES, I. I. B.; VASCONCELOS, K. C. de; SILVA, L. L. L. Análise da qualidade de vida segundo o Questionário SF-36 nos funcionários da gerência de assistência nutricional (GAN) da Fundação Santa Casa de Misericórdia do Pará. Belém, 2009, 76 f. Dissertação (Bacharelado em Fisioterapia). Universidade da Amazônia, Belém, 2009.

FLORINI, C. A. Cuidador familiar: sobrecarga e proteção. Revista Brasileira de Cancerologia, v. 50, n. 4, p. 341-345, 2004. Disponível em: <http://www1.inca.gov.br/rbc/n_50/v04/pdf/secao5.pdf>. Acesso em: 05 dez. 2016.

PAGANINI, D. D. Síndrome de Burnout. Criciúma, 2011, 50 f. Monografia (Especialização em Engenharia de Segurança do Trabalho). Universidade do Extremo Sul Catarinense - UNESC, Criciúma, 2011.

PECHORRO, P.; MAROCÔ, J.; POIARES, C.; VIEIRA, R. X. Validação da Escala de Auto-Estima de Rosenberg com Adolescentes Portugueses em Contexto Forense e Escolar. Arquivos de Medicina, Porto, v. 25, n. 5, p. 174-179, 2011. Disponível em: <http://www.scielo.mec.pt/pdf/am/v25n5-6/v25n5-6a02.pdf>. Acesso em: 20 nov. 2016.

SCHNAIDER, T. B.; SILVA, J. B.; PEREIRA, M. A. R. Cuidador Familiar de Paciente com Afecção Neurológica. Saúde e Sociedade, São Paulo, v. 18, n. 2, p. 284-292, 2009. Disponível em: <http://www.scielo.br/pdf/ sausoc/v18n2/11.pdf>. Acesso em: 20 nov. 2016.

SILVA, M. J.; BRAGA, M. M.; SILVA, B. C. T. Avaliação da presença da síndrome de Burnout em cuidadores de idosos. Enfermería Global, v. 8, n. 2, jun. 2009. Disponível em: <http://revistas.um.es/eglobal/article/ view/65451/66881>. Acesso em: 15 nov. 2016.

TSUKAMOTO, H. F.; PICINATTO, A. E.; CAVALINI, C. A.; BORTOLLOTI, L. F. Análise de independência funcional, qualidade de vida, força muscular respiratória e mobilidade torácica em pacientes hemiparéticos submetidos a um programa de reabilitação: estudos de caso. Semina: Ciências Biológicas da Saúde, Londrina, v. 31, n. 1, p. 63-69, jan./jun. 2010. Disponível em: <http://dx.doi.org/10.5433/1679-0367.2010v31n1p63>. Acesso em: 15 nov. 2016.

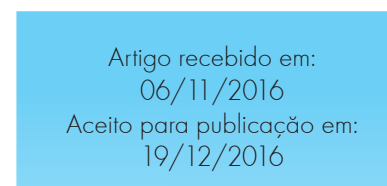




\section{ANEXOS}

\section{TERMO DE CONSENTIMENTO LIVRE E ESCLARECIDO}

\section{"Avaliaçáo da Qualidade de Vida e Presença da Síndrome de Burnout em Cuidadores de Pacientes Neurológicos Antes e Após um Programa de Orientaçóes"}

Prezado(a) Senhor(a):

Gostaríamos de convidá-lo (a) para participar da pesquisa "Avaliaçáo da Qualidade de Vida e Presença da Síndrome de Burnout em Cuidadores de Pacientes Neurológicos Antes e Após um Programa de Orientaçóes", a ser realizada na Universidade Estadual do Norte do Paraná - Jacarezinho/PR. O objetivo da pesquisa é "Avaliar a qualidade de vida e a presença da Sindrome de Burnout de cuidadores (familiares) de pacientes neurológicos". Sua participaçáo é muito importante e ela se daria da seguinte forma: Durante a realização da pesquisa, haverá dois momentos de avaliaçóes através de escalas e questionários, as quais acontecerão uma no início e outra no fim da pesquisa, entre esses dois momentos será realizado um programa de orientaçóes individuais, que terá como objetivo sanar as dúvidas existentes e acrescentar conhecimento em relação aos cuidados prestados aos pacientes.

Esclarecemos que sua participação é totalmente voluntária, podendo o(a) senhor(a) recusar-se a participar, ou mesmo desistir a qualquer momento, sem que isto acarrete qualquer ônus ou prejuízo à sua pessoa. Esclarecemos, também, que suas informaçôes serão utilizadas somente para os fins desta pesquisa e seráo tratadas com o mais absoluto sigilo e confidencialidade, de modo a preservar a sua identidade.

Esclarecemos ainda, que o(a) senhor(a) não pagará e nem será remunerado(a) por sua participaçáo. Garantimos, no entanto, que todas as despesas decorrentes da pesquisa seráo ressarcidas, quando devidas e decorrentes especificamente de sua participação.

Os benefícios esperados com a pesquisa são, a partir dos dados científicos, oferecer alternativas clínicas para futuros tratamentos com enfoque em funcionalidade e qualidade de vida.

Quanto aos riscos, pode acontecer de o paciente sofrer alguma lesão durante as transferências (um dos assuntos abordado no programa de orientaçóes), por este motivo o mesmo estará sendo monitorado cuidadosamente pelo avaliador e quando possível existirá um segundo avaliador oferecendo suporte ao paciente.

Caso o(a) senhor(a) tenha dúvidas ou necessite de maiores esclarecimentos poderá nos contatar através das seguintes informaçôes: Joyce Karla Machado da Silva, rua Alameda Padre Magno 841, Bairro Nova Jacarezinho, CEP: 86400-000, Jacarezinho - PR. Telefone profissional: (43) 3525-0498, celular: (43) 9981-3363, e-mail: jksilva@uenp.edu.br.

Este termo deverá ser preenchido em duas vias de igual teor, sendo uma delas devidamente preenchida, assinada e entregue ao(à) senhor(a).

$$
\text { Jacarezinho, __ de ___ de } 2016 .
$$

tendo sido devidamente esclarecido sobre os procedimentos da pesquisa, concordo em participar voluntariamente da pesquisa descrita acima.

Assinatura (ou impressáo dactiloscópica):

Data: 
1- Em geral você diria que sua saúde é:

\begin{tabular}{|c|c|c|c|c|}
\hline Excelente & Muito boa & Boa & Ruim & Muito ruim \\
\hline 1 & 2 & 3 & 4 & 5 \\
\hline
\end{tabular}

2- Em comparação há um ano, como você classificaria sua idade em geral, agora?

\begin{tabular}{|c|c|c|c|c|}
\hline Muito melhor & Um pouco melhor & Quase a mesma & Um pouco pior & Muito pior \\
\hline 1 & 2 & 3 & 4 & 5 \\
\hline
\end{tabular}

3- Os seguintes itens são sobre atividades que você poderia fazer atualmente durante um dia comum. Devido à sua saúde, você teria dificuldade para fazer estas atividades? Neste caso, quando?

\begin{tabular}{|l|c|c|c|}
\hline \multicolumn{1}{|c|}{ Atividades } & $\begin{array}{c}\text { Sim, dificulta } \\
\text { muito }\end{array}$ & $\begin{array}{c}\text { Sim, dificulta um } \\
\text { pouco }\end{array}$ & $\begin{array}{c}\text { Não, não dificulta } \\
\text { de modo algum }\end{array}$ \\
\hline $\begin{array}{l}\text { a) Atividades rigorosas, que exigem muito } \\
\text { esforço, tais como correr, levantar objetos } \\
\text { pesados, participar em esportes árduos. }\end{array}$ & 1 & 2 & 3 \\
\hline $\begin{array}{l}\text { b) Atividades moderadas, tais como mover uma } \\
\text { mesa, passar aspirador de pó, jogar bola, varrer } \\
\text { a casa. }\end{array}$ & 1 & 2 & 3 \\
\hline c) Levantar ou carregar mantimentos & 1 & 2 & 3 \\
\hline d) Subir vários lances de escada & 1 & 2 & 3 \\
\hline e) Subir um lance de escada & 1 & 2 & 3 \\
\hline f) Curvar-se, ajoelhar-se ou dobrar-se & 1 & 2 & 3 \\
\hline g) Andar mais de 1 quilômetro & 1 & 2 & 3 \\
\hline h) Andar vários quarteirões & 1 & 2 & 3 \\
\hline i) Andar um quarteirão & 1 & 2 & 3 \\
\hline j) Tomar banho ou vestir-se & 1 & 2 & \\
\hline
\end{tabular}

4- Durante as últimas 4 semanas, você teve algum dos seguintes problemas com seu trabalho ou com alguma atividade regular, como consequência de sua saúde física?

\begin{tabular}{|l|c|c|}
\hline & Sim & Não \\
\hline $\begin{array}{l}\text { a) Você diminui a quantidade de tempo que se dedicava ao seu trabalho ou a } \\
\text { outras atividades? }\end{array}$ & 1 & 2 \\
\hline b) Realizou menos tarefas do que você gostaria? & 1 & 2 \\
\hline c) Esteve limitado no seu tipo de trabalho ou a outras atividades. & 1 & 2 \\
\hline $\begin{array}{l}\text { d) Teve dificuldade de fazer seu trabalho ou outras atividades (por exemplo, ne- } \\
\text { cessitou de um esforço extra). }\end{array}$ & 1 & 2 \\
\hline
\end{tabular}

5- Durante as últimas 4 semanas, você teve algum dos seguintes problemas com seu trabalho ou outra atividade regular diária, como consequência de algum problema emocional (como se sentir deprimido ou ansioso)?

\begin{tabular}{|l|c|c|}
\hline & Sim & Não \\
\hline $\begin{array}{l}\text { a) Você diminui a quantidade de tempo que se dedicava ao seu trabalho ou a } \\
\text { outras atividades? }\end{array}$ & 1 & 2 \\
\hline b) Realizou menos tarefas do que você gostaria? & 1 & 2 \\
\hline $\begin{array}{l}\text { c) Não realizou ou fez qualquer das atividades com tanto cuidado, como } \\
\text { geralmente faz. }\end{array}$ & 1 & 2 \\
\hline
\end{tabular}


6- Durante as últimas 4 semanas, de que maneira sua saúde física ou problemas emocionais interferiram nas suas atividades sociais normais, em relação à família, amigos ou em grupo?

\begin{tabular}{|c|c|c|c|c|}
\hline De forma nenhuma & Ligeiramente & Moderadamente & Bastante & Extremamente \\
\hline 1 & 2 & 3 & 4 & 5 \\
\hline
\end{tabular}

7- Quanta dor no corpo você teve durante as últimas 4 semanas?

\begin{tabular}{|c|c|c|c|c|c|}
\hline Nenhuma & Muito leve & Leve & Moderada & Grave & Muito grave \\
\hline 1 & 2 & 3 & 4 & 5 & 6 \\
\hline
\end{tabular}

8- Durante as últimas 4 semanas, quanto a dor interferiu com seu trabalho normal (incluindo o trabalho dentro de casa)?

\begin{tabular}{|c|c|c|c|c|}
\hline De maneira alguma & Um pouco & Moderadamente & Bastante & Extremamente \\
\hline 1 & 2 & 3 & 4 & 5 \\
\hline
\end{tabular}

9- Estas questóes são sobre como você se sente e como tudo tem acontecido com você durante as últimas 4 semanas. Para cada questáo, por favor, dê uma resposta que mais se aproxime da maneira como você se sente em relação às últimas 4 semanas.

\begin{tabular}{|l|c|c|c|c|c|c|}
\hline & $\begin{array}{c}\text { Todo } \\
\text { Tempo }\end{array}$ & $\begin{array}{c}\text { A maior } \\
\text { parte do } \\
\text { tempo }\end{array}$ & $\begin{array}{c}\text { Uma boa } \\
\text { parte do } \\
\text { tempo }\end{array}$ & $\begin{array}{c}\text { Alguma parte } \\
\text { do tempo }\end{array}$ & $\begin{array}{c}\text { Uma pequena } \\
\text { parte do } \\
\text { tempo }\end{array}$ & Nunca \\
\hline $\begin{array}{l}\text { a) Quanto tempo você tem se } \\
\text { sentindo cheio de vigor, de } \\
\text { vontade, de força? }\end{array}$ & 1 & 2 & 3 & 4 & 5 & 6 \\
\hline $\begin{array}{l}\text { b) Quanto tempo você tem } \\
\text { se sentido uma pessoa muito } \\
\text { nervosa? }\end{array}$ & 1 & 2 & 3 & 4 & 5 & 6 \\
\hline $\begin{array}{l}\text { c) Quanto tempo você tem } \\
\text { se sentido tão deprimido que } \\
\text { nada pode animá-lo? }\end{array}$ & 1 & 2 & 3 & 4 & 5 & 6 \\
\hline $\begin{array}{l}\text { d) Quanto tempo você tem se } \\
\text { sentido calmo ou tranquilo? }\end{array}$ & 1 & 2 & 3 & 4 & 5 & 6 \\
\hline $\begin{array}{l}\text { e) Quanto tempo você tem se } \\
\text { sentido com muita energia? }\end{array}$ & 1 & 2 & 3 & 4 & 5 & 6 \\
\hline $\begin{array}{l}\text { f) Quanto tempo você tem } \\
\text { se sentido desanimado ou } \\
\text { abatido? }\end{array}$ & 1 & 2 & 3 & 4 & 5 & 6 \\
\hline $\begin{array}{l}\text { g) Quanto tempo você tem se } \\
\text { sentido esgotado? }\end{array}$ & 1 & 2 & 3 & 4 & 5 & 6 \\
\hline $\begin{array}{l}\text { h) Quanto tempo você tem se } \\
\text { sentido uma pessoa feliz? }\end{array}$ & 1 & 2 & 3 & 4 & 5 & 6 \\
\hline $\begin{array}{l}\text { i) Quanto tempo você tem se } \\
\text { sentido cansado? }\end{array}$ & 1 & 2 & 3 & 4 & 5 & 6 \\
\hline
\end{tabular}

10- Durante as últimas 4 semanas, quanto de seu tempo a sua saúde física ou problemas emocionais interferiram com as suas atividades sociais (como visitar amigos, parentes etc)?

\begin{tabular}{|c|c|c|c|c|}
\hline Todo Tempo & $\begin{array}{c}\text { A maior parte do } \\
\text { tempo }\end{array}$ & Alguma parte do tempo & $\begin{array}{c}\text { Uma pequena parte } \\
\text { do tempo }\end{array}$ & $\begin{array}{c}\text { Nenhuma parte do } \\
\text { tempo }\end{array}$ \\
\hline 1 & 2 & 3 & 4 & 5 \\
\hline
\end{tabular}


11- O quanto verdadeiro ou falso é cada uma das afirmaçóes para você?

\begin{tabular}{|l|c|c|c|c|c|}
\hline & $\begin{array}{c}\text { Definitivamente } \\
\text { verdadeiro }\end{array}$ & $\begin{array}{c}\text { A maioria } \\
\text { das vezes } \\
\text { verdadeiro }\end{array}$ & Não sei & $\begin{array}{c}\text { A maioria } \\
\text { das vezes } \\
\text { falso }\end{array}$ & $\begin{array}{c}\text { Definitivamente } \\
\text { falso }\end{array}$ \\
\hline $\begin{array}{l}\text { a) Eu costumo obedecer um } \\
\text { pouco mais facilmente que as } \\
\text { outras pessoas }\end{array}$ & 1 & 2 & 3 & 4 & 5 \\
\hline $\begin{array}{l}\text { b) Eu sou tão saudável quanto } \\
\text { qualquer pessoa que eu } \\
\text { conheço }\end{array}$ & 1 & 2 & 3 & 4 & 5 \\
\hline $\begin{array}{l}\text { c) Eu acho que a minha saúde } \\
\text { vai piorar }\end{array}$ & 1 & 2 & 3 & 4 & 5 \\
\hline d) Minha saúde é excelente & 1 & 2 & 3 & 4 & 5 \\
\hline
\end{tabular}

\section{QUESTIONÁRIO PRELIMINAR DE IDENTIFICAÇÃO DA BURNOUT}

Obs.: este instrumento é de uso informativo apenas e não deve substituir o diagnóstico realizado por médico ou psicoterapeuta.

MARQUE "X" na coluna correspondente:

Nunca |2-Anualmente |3-Mensalmente |4-Semanalmente |5-Diariamente

\begin{tabular}{|c|c|c|c|c|c|c|}
\hline $\mathbf{N}^{\circ}$ & Características psicofísicas em relação ao trabalho & 1 & 2 & 3 & 4 & 5 \\
\hline 1 & Sinto-me esgotado(a) emocionalmente em relação ao meu trabalho & & & & & \\
\hline 2 & Sinto-me excessivamente exausto ao final da minha jornada de trabalho & & & & & \\
\hline 3 & Levanto-me cansado(a) e sem disposição para realizar o meu trabalho & & & & & \\
\hline 4 & Envolvo-me com facilidade nos problemas dos outros & & & & & \\
\hline 5 & Trato algumas pessoas como se fossem da minha família & & & & & \\
\hline 6 & Tenho que desprender grande esforço para realizar minhas tarefas laborais & & & & & \\
\hline 7 & Acredito que eu poderia fazer mais pelas pessoas assistidas por mim & & & & & \\
\hline 8 & Sinto que meu salário é desproporcional às funções que executo & & & & & \\
\hline 9 & Sinto que sou uma referência para as pessoas que lido diariamente & & & & & \\
\hline 10 & Sinto-me com pouca vitalidade, desanimado(a) & & & & & \\
\hline 11 & Não me sinto realizado(a) com o meu trabalho & & & & & \\
\hline 12 & Não sinto mais tanto amor pelo meu trabalho como antes & & & & & \\
\hline 13 & Não acredito mais naquilo que realizo profissionalmente & & & & & \\
\hline 14 & Sinto-me sem forças para conseguir algum resultado significante & & & & & \\
\hline 15 & Sinto que estou no emprego apenas por causa do salário & & & & & \\
\hline 16 & Tenho me sentido mais estressado(a) com as pessoas que atendo & & & & & \\
\hline 17 & Sinto-me responsável pelos problemas das pessoas que atendo & & & & & \\
\hline 18 & Sinto que as pessoas me culpam pelos seus problemas & & & & & \\
\hline 19 & $\begin{array}{l}\text { Penso que não importa o que eu faça, pois nada vai mudar no meu } \\
\text { trabalho }\end{array}$ & & & & & \\
\hline 20 & Sinto que não acredito mais na profissão que exerço & & & & & \\
\hline \multicolumn{2}{|r|}{ Totais (multiplique o número de $\mathrm{X}$ pelo valor da coluna) } & & & & & \\
\hline & Some o total de cada coluna e obtenha seu score & & & & & \\
\hline
\end{tabular}


ESCALA DE AUTOESTIMA DE ROSENBERG - RSES

Eu sinto que sou uma pessoa de valor, no mínimo, tanto quanto as outras pessoas.

(1) Discordo totalmente (2) Discordo (3) Concordo (4) Concordo totalmente

Eu acho que eu tenho várias boas qualidades.

(1) Discordo totalmente (2) Discordo (3) Concordo (4) Concordo totalmente

Levando tudo em conta, eu penso que eu sou um fracasso.

(1) Discordo totalmente (2) Discordo (3) Concordo (4) Concordo totalmente

Eu acho que sou capaz de fazer as coisas tâo bem quanto a maioria das pessoas.

(1) Discordo totalmente (2) Discordo (3) Concordo (4) Concordo totalmente

Eu acho que não tenho muito do que me orgulhar.

(1) Discordo totalmente (2) Discordo (3) Concordo (4) Concordo totalmente

Eu tenho uma atitude positiva em relação a mim mesmo.

(1) Discordo totalmente (2) Discordo (3) Concordo (4) Concordo totalmente

No conjunto, eu estou satisfeito comigo.

(1) Discordo totalmente (2) Discordo (3) Concordo (4) Concordo totalmente

Eu gostaria de poder ter mais respeito comigo mesmo.

(1) Discordo totalmente (2) Discordo (3) Concordo (4) Concordo totalmente 
Às vezes eu me sinto inútil.

(1) Discordo totalmente (2) Discordo (3) Concordo (4) Concordo totalmente

10) Às vezes eu acho que náo presto pra nada.

(1) Discordo totalmente (2) Discordo (3) Concordo (4) Concordo totalmente 